\title{
Beam-beam interaction effects for separated beams in a proton-antiproton collider
}

\author{
D. Siergiej \\ University of New Mexico, Albuquerque, New Mexico 87131 \\ D. Finley \\ Fermi National Accelerator Laboratory, Batavia, Illinois 60510
}

W. Herr

European Laboratory for Particle Physics (CERN), Geneva, Switzerland

(Received 31 July 1996; revised manuscript received 11 November 1996)

\begin{abstract}
An investigation of the beam-beam interaction as a function of transverse separation of colliding proton and antiproton bunches is presented. A mathematical model describing resonant excitation of separated beams is examined. Beam-beam experiments in the Fermilab collider measure beam-beam resonant excitation (particle losses) at different transverse beam separations in the storage ring. The experimental results are compared to simulated particle losses in a beam-beam simulation model. [S1063-651X(97)00503-5]
\end{abstract}

PACS number(s): 41.85.-p, 29.27.Bd

\section{INTRODUCTION}

The electromagnetic interaction between two colliding particle distributions (the beam-beam interaction) has in the past been a dominant factor in limiting the integrated luminosity in a colliding beam storage ring [1]. Efforts to curb this measurable luminosity limitation in the Fermilab collider led to an implementation of a helical orbit scheme. Proton and antiproton orbits were separated in both transverse planes at every beam-beam collision point except for the two crossing points at the high energy physics detectors. An investigation of beam-beam interaction effects of colliding proton and antiproton distributions which are separated transversely is presented in this paper.

A weak-strong model of the beam-beam interaction is used to describe the motion of a "weak" or low intensity antiproton bunch colliding with a "strong" or high intensity proton bunch. Antiprotons, in a weak-strong model, are the main focus of attention as test particles. Test particles differ from each other in amplitude.

It is of interest to examine a particle's motion under a single resonant excitation. The resonant driving term of the beam-beam interaction is nearly isolated when an antiproton's tune $Q$ is close to a value satisfying $n Q=p$, where $n$ and $p$ are integers. A convenient method of isolating the beam-beam resonant driving term is to use a Fourier series expansion of the beam-beam potential. In one dimension, the Fourier series expansion of the beam-beam potential in action-angle coordinates $(J, \phi)$ is

$$
V(J, \phi)=\sum_{n=1}^{\infty} V_{n}(J) \cos n \phi,
$$

where the summation over $n$ includes all orders of resonances. In an isolated resonance model, a single resonance of order $n$ is accepted as the dominant resonant excitation term. The resonant width function, $V_{n}(J)$, represents the amplitude of the dominant resonant excitation term of the beam-beam potential [2]. The beam-beam resonant excitation for a given resonance is obtained by taking the derivative of the beambeam potential with respect to the action coordinate. The resultant beam-beam resonant driving term for a resonance of order $n$ is

$$
\begin{aligned}
V_{n}^{\prime}(\alpha)= & \xi \frac{1}{2 \pi} \int_{0}^{2 \pi} d \phi \frac{\cos n \phi \cos \phi}{\alpha\left(\cos \phi-\frac{d_{N}}{2 \sqrt{\alpha}}\right)} \\
& \times\left\{1-\exp \left[-2\left(\sqrt{\alpha} \cos \phi-\frac{d_{N}}{2}\right)^{2}\right]\right\},
\end{aligned}
$$

where $\alpha=\sqrt{2 \beta J}$ ( $\beta$ is the beta function at the collision point), $\xi$ is the beam-beam tuneshift parameter, and the prime denotes a derivative with respect to $J$ [3].

Beam separation is defined as the distance between the orbit of a zero amplitude antiproton and the orbit of the centroid of the colliding proton distribution. The parameter $d_{N}$ denotes the transverse beam separation $d$ normalized to the rms transverse size of the proton distribution $\sigma$; $d_{N}=d / \sigma$. Units of beam separation are expressed in terms of $\sigma$.

For head-on collisions $(d=0)$, the amplitude of beambeam resonant excitation for various even-ordered resonances is shown in Fig. 1. Note that the absolute magnitude of resonant excitation is greater for lower order resonances than for higher order resonances. This is true for a particle at any oscillation amplitude.

Resonant excitation for a colliding beam separation of 2 $\sigma$ is shown in Fig. 2. As seen in the figure, a nonzero beam separation introduces resonant components of the beambeam force which drive odd-ordered resonances [2]. It is apparent that for separated beams, one can no longer generalize that the amplitude of resonant excitation is larger for lower order resonances. A particle oscillating at a given amplitude may see a larger resonance excitation near a higher order resonance. 


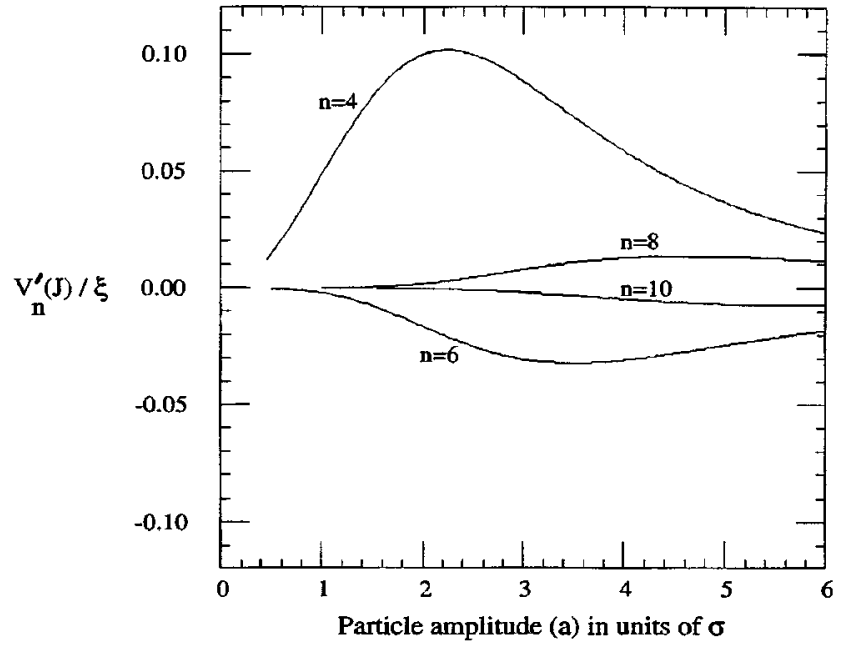

FIG. 1. Amplitude of a beam-beam driven resonance of order $n$ for head-on collisions. A round beam approximation is used.

The amplitude of resonant excitation due to the beambeam interaction for various beam separations is shown in Fig. 3. It is also apparent in this figure that it is difficult to predict how destructive a resonance will be on a particle distribution at different beam separations.

The mathematical model, therefore, does not lead to any easy generalizations which can be used to predict colliding beam behavior in the presence of a nonzero separation. For this reason, a simulation code was developed to simulate beam-beam collisions in the Fermilab collider. Simulation results are compared to experimental measurements of beambeam resonant excitation in the Fermilab collider.

\section{BEAM-BEAM EXPERIMENTS IN THE FERMILAB COLLIDER}

Table I lists typical values of the experimental parameters and the accelerator lattice parameters for the collider experi-

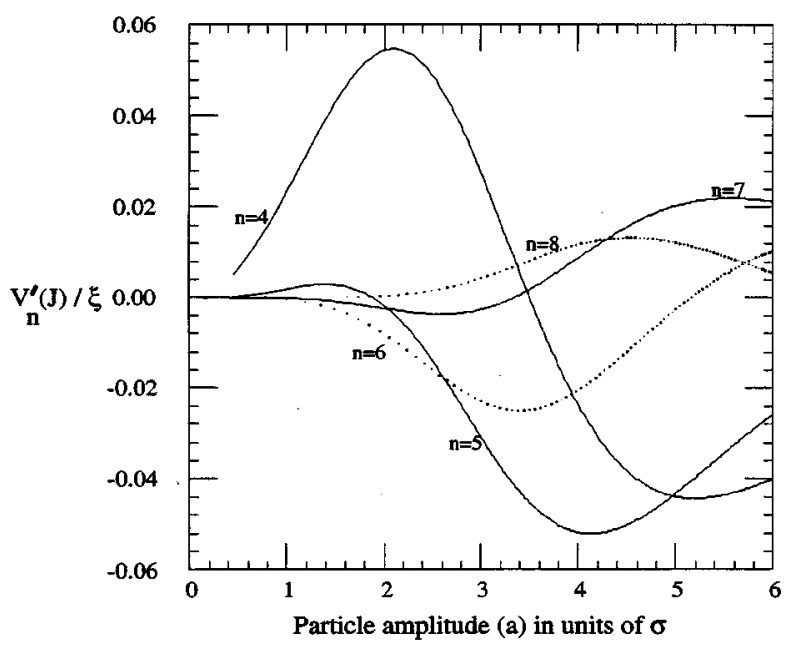

FIG. 2. Amplitude of a beam-beam driven resonance of order $n$ for a beam separation of $2 \sigma$. A round beam approximation is used.

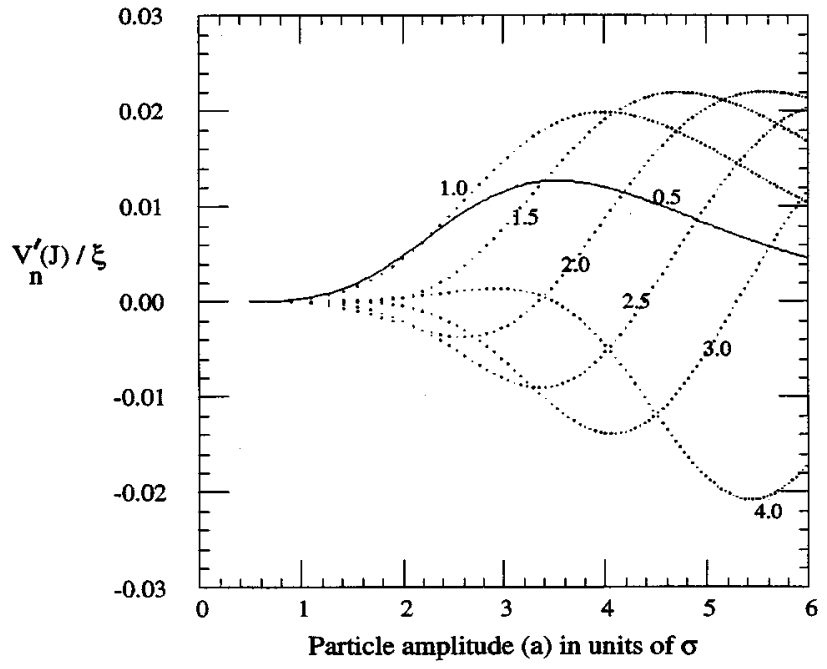

FIG. 3. Amplitude of a beam-beam driven resonance of seventh order for various horizontal separations in units of $\sigma$. A round beam approximation is used.

ments discussed below. The values listed for beam emittance refer to $95 \%$ normalized emittance values; $95 \%$ of the particle distribution in phase space is included in the definition of beam size.

Control of the beam separation and crossing angle for all experiments was obtained using separator four bumps [4]. The accuracy in measuring the beam separation and the crossing angle is determined by measuring the luminosity as the beam separation and crossing angle are varied. Luminosity as a function of separation and crossing angle, along with a fit to the data, is shown in Figs. 4 and 5, respectively. The standard deviation of the Gaussian fit in Fig. 4 is the convolution of individual proton and antiproton widths; $\sqrt{{\sigma_{p_{y}}}^{2}+\sigma_{\bar{p}_{y}}{ }^{2}}$ [4]. Assuming equal beam sizes, the accuracy of beam separation is estimated from the accuracy in the standard deviation obtained from the Gaussian fit. From Fig. 4 , the accuracy in beam separation is thus estimated to be known within $0.05 \sigma$.

TABLE I. Experimental parameters.

\begin{tabular}{lc}
\hline \hline Accelerator parameters & Value \\
\hline Kinetic energy $(\mathrm{GeV})$ & 900 \\
Beam-beam tune shift parameter & 0.005 \\
Beta at B0 collision point & \\
Horizontal beta $(\mathrm{m})$ & 0.35 \\
Vertical beta $(\mathrm{m})$ & 0.35 \\
Horizontal dispersion $(\mathrm{m})$ & -0.005 \\
Slope of dispersion $(\mathrm{m})$ & 0.28 \\
Synchrotron tune & $5.7 \times 10^{-4}$ \\
Momentum spread & $1 \times 10^{-4}$ \\
Protons per bunch & $200 \times 10^{9}$ \\
Antiprotons per bunch & $70 \times 10^{9}$ \\
Transverse emittance $(\pi \mathrm{mm} \mathrm{mrad})$ & \\
Proton & 25 \\
Antiproton & 15 \\
Longitudinal emittance $(\mathrm{eV} \mathrm{sec})$ & 4 \\
\hline \hline
\end{tabular}




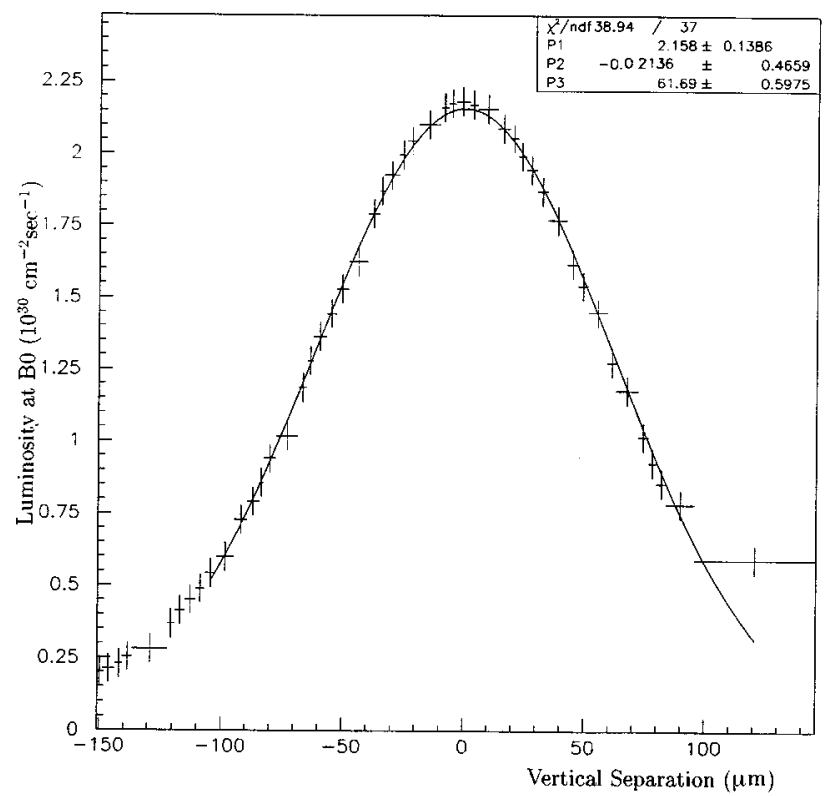

FIG. 4. A MINUIT fit of measured luminosity vs vertical beam separation of proton-antiproton collisions at $B 0$.

\section{A. Identification of beam-beam driven resonances}

Measured proton and antiproton tunes in the collider are nominally in an area in betatron tune space that border 7th and 5th order resonances. Under separated beam conditions, a measure of proton losses during tune scans were used to identify whether these odd-ordered resonances were beambeam driven resonances. Figure 6 compares measured proton losses as the proton tune is moved across 5th order resonances for a proton only store of six bunches and a $6 \times 6$ colliding beam store. It is evident from the measured losses that the 5 th order resonance is driven both by the collider lattice itself and by the beam-beam interaction between col-

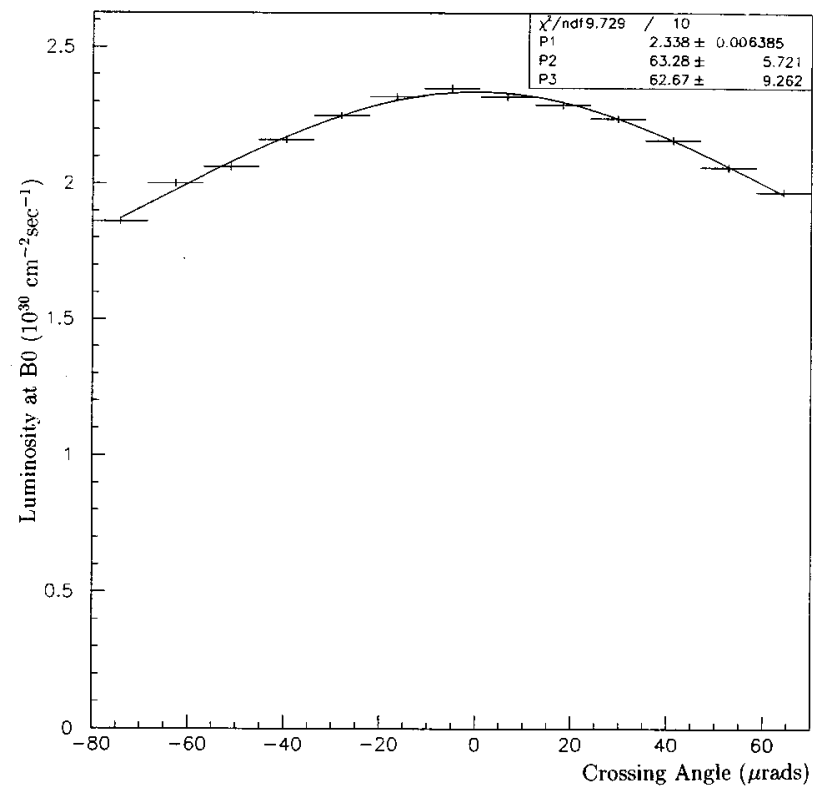

FIG. 5. A MINUIT fit of measured luminosity vs horizontal crossing angle of proton-antiproton collisions at $B 0$.

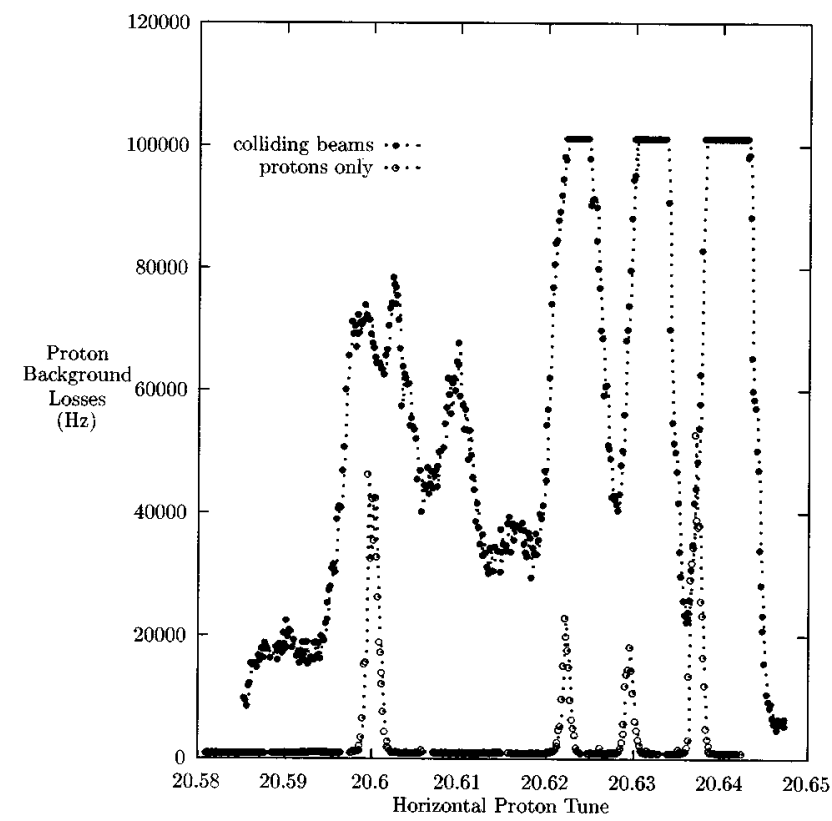

FIG. 6. A comparison of proton losses measured at $B 0$ while crossing fifth order resonances for protons only and a colliding beam store. The tune scan was "diagonal" in that the vertical tune was also varied during the tune scan from 20.545 to 20.645 .

liding protons and antiprotons. Proton losses are seen to be significant only in the case of tune scans with colliding beams when crossing 7 th order resonances in Fig. 7. From these measurements, it is concluded that the beam-beam interaction is the sole driving term which at least initially drives 7th order resonances in the collider. Once a particle's amplitude grows due to nonlinearities of the beam-beam interaction, it can be lost because of nonlinear kicks it receives from elements in the collider lattice itself.

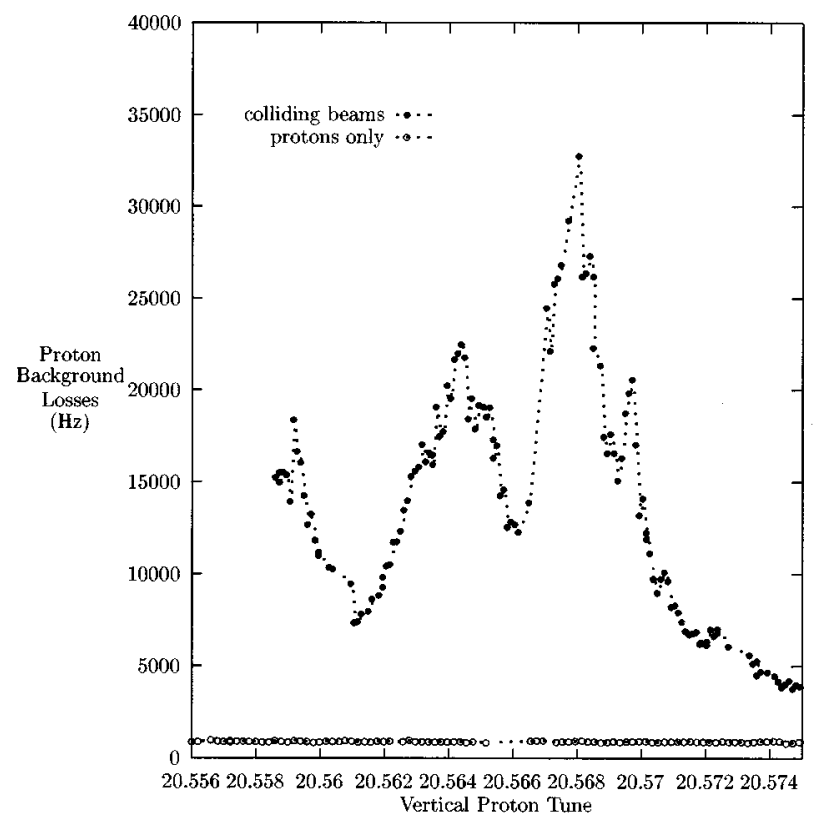

FIG. 7. A comparison of proton losses measured at $B 0$ while crossing seventh order resonances for protons only and a colliding beam store. The horizontal tune remained constant at 20.585 . 


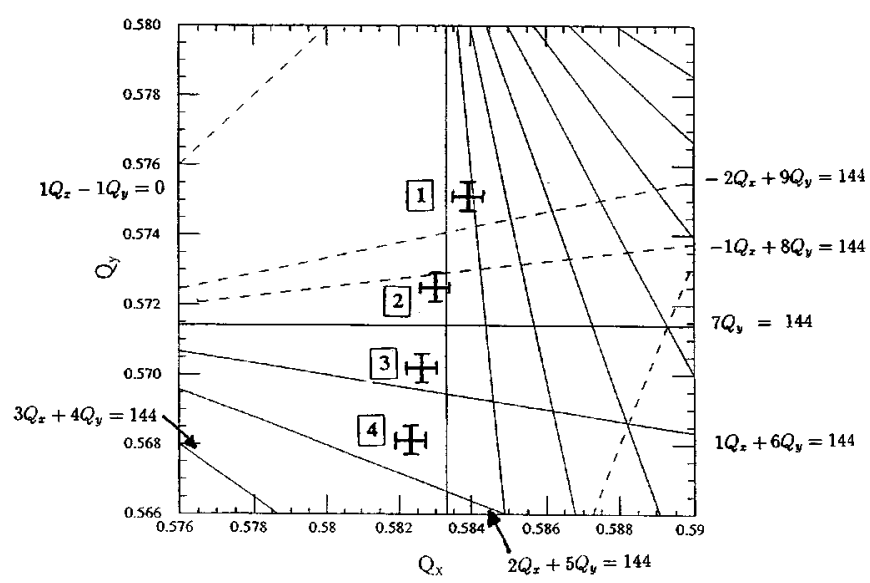

FIG. 8. Locations in tune space in which resonant effects were measured. The measured proton tune along with its uncertainty is depicted.

\section{B. Resonant effects as a function of transverse beam separation}

Measurements of beam-beam interaction effects as a function of beam separation were done using a $1 \times 1$ colliding beam store; one proton bunch colliding with one antiproton bunch at two locations in the storage ring. The two bunches were set to collide at the $B O$ high energy physics detector and consequently also collided at the opposing EO location in the collider. In the experiment, the separation of colliding protons and antiprotons was varied at $B 0$ while the separation at $E 0$ remained constant at $4 \sigma$. Particle losses were measured at four locations in tune space, as labeled in Fig. 8. The uncertainty in the measured proton tune at each location is represented in the figure. This uncertainty in tune is the standard deviation of four tune measurements taken for four different beam separations at the same proton tune settings, or equivalently, correction quadrupole current settings. There exists a transient behavior of particle losses during a tune change, therefore measurements of particle losses were taken only after losses reached an equilibrium value after a tune change [4]. Fig. 9 represents particle losses at each labeled tune location under conditions of four different transverse beam separations; the orbits were separated equally in each plane by $0 \sigma, 1 \sigma, 2 \sigma$, and $3 \sigma$. The rms beam separation was thus $0 \sigma, 1.4 \sigma, 2.8 \sigma$, and $4.2 \sigma$, respectively.

Resonant effects at each tune location are assumed to be related to the measured antiproton losses. From Fig. 9, antiproton losses are minimal for head-on collisions in all cases; this result is expected in a region of odd-ordered resonances. At the tune setting of measurement 1 in Fig. 8, antiproton losses are minimal for both head-on collisions and for separated beam conditions. This is the operating tune for colliding beam stores in the collider. No beam-beam driving terms are observed to strongly drive 9th or 11th order difference resonances. The presence of beam separation is seen to excite odd-ordered sum resonances at the tune locations of measurements 2 and 3 . In measurement 2 , the largest resonant effects are observed at an rms separation of $2.8 \sigma$ separation. In measurement 3 , resonant effects are largest at $4.2 \sigma$ separation.

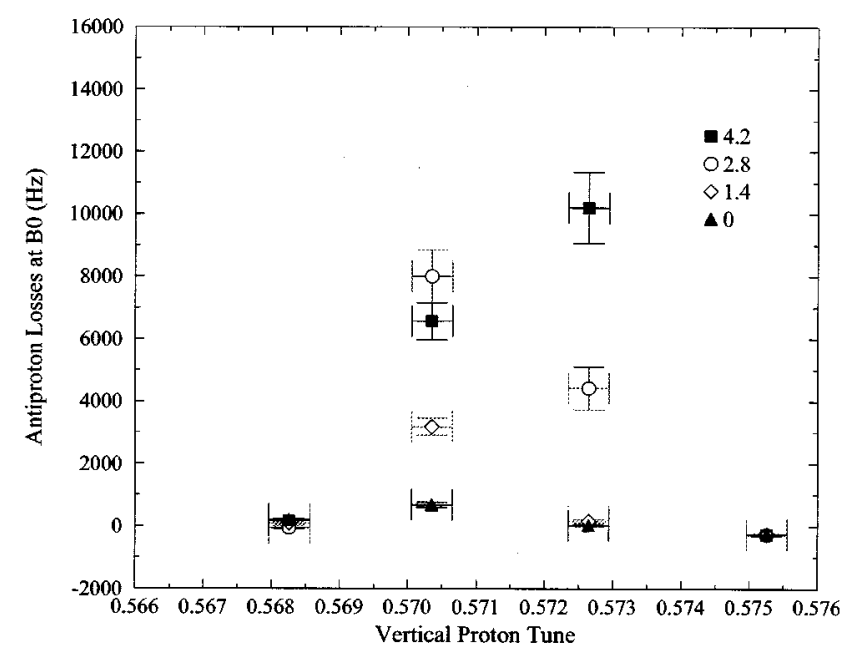

FIG. 9. Antiproton background losses at $B 0$ when the proton tune is near 7th, 9th, and 11th order resonances. Each symbol represents a different proton-antiproton bunch separation at $B 0$ in units of $\sigma$.

\section{RESONANT EFFECTS MEASURED IN A BEAM-BEAM SIMULATION}

The simulation code developed to simulate resonant effects in the Fermilab collider was based on a previously developed code [5].

The model used for the simulation is concerned only with particle motion due to the beam-beam interaction. The motion of a particle between beam-beam crossing points is assumed to be linear motion. The particle experiences an angular kick due to the beam-beam interaction at each beambeam crossing point. Beam-beam kicks of magnitude $\Delta x^{\prime}$ and $\Delta y^{\prime}$ are calculated for nonround beams in the simulation. A vertical kick in the simulation is given by

$$
\Delta y^{\prime}=-\frac{2 N_{b} r_{p} y}{\gamma}\left(\frac{2 \pi}{a^{2}-b^{2}}\right)^{1 / 2} \operatorname{Re}[f(x, y)]
$$

where

$$
\begin{aligned}
f(x, y)= & w\left(\frac{x+i y}{\sqrt{2\left(a^{2}-b^{2}\right)}}\right) \\
& -\exp \left[\left(-\frac{x^{2}}{2 a^{2}}-\frac{y^{2}}{2 b^{2}}\right)\right] w\left(\frac{x \frac{b}{a}+i y \frac{a}{b}}{\sqrt{2\left(a^{2}-b^{2}\right)}}\right),
\end{aligned}
$$

for $a>b[6,7]$. The parameters $a$ and $b$ denote the horizontal and vertical bunch sizes of the colliding proton distribution. The function $w(A+i B)$ is the complex error function. The real part of the square brackets of Eq. (3.1) is used to calculate the vertical kick and the imaginary part is used to calculate the horizontal kick. 

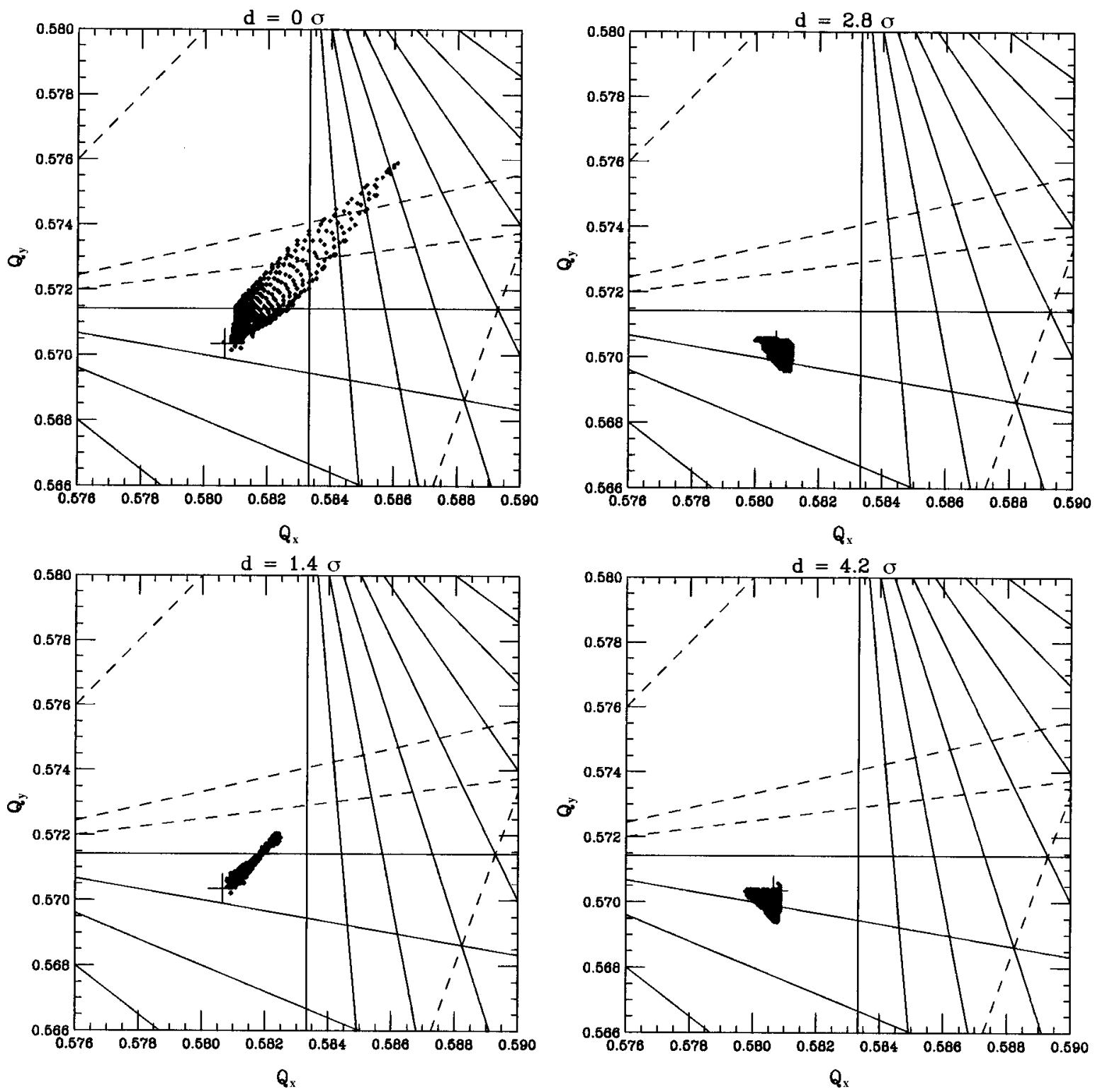

FIG. 10. Antiproton beam-beam tune shift due to beam-beam detuning overlaid on the tune of measurement 2 . Tune shifts due to resonant effects are not shown. The proton intensity is $120 \times 10^{9}$. Beam-beam footprints represent collision points at both $B 0$ and $E 0$. Each plot represents a different beam separation at $B 0$.

The beam-beam interaction does depend upon the longitudinal position of a particle. Synchro-betatron resonances are excited when a nonzero crossing angle is present $[8,9]$. However, beam-beam simulations and experiments done in the super proton synchrotron collider demonstrated beam stability in the presence of large crossing angles [10]. Since the beam-beam collisions considered in this paper are separated and have a zero crossing angle, longitudinal motion is not included in the beam-beam kick expression.

In order to calculate a particle's tune $q$ in the presence of a nonzero beam separation, it is necessary to calculate the gradient of the beam-beam kick around the closed orbit of the particle;

$$
q \propto \frac{\partial\left(\Delta x^{\prime}\right)}{\partial x}
$$

When Eq. (3.3) is evaluated for zero-amplitude particles, a dipole kick is apparent. The dipole kick is a constant orbit kick which is independent of particle amplitude. A shift in a particle's closed orbit occurs due to this dipole kick. The tune of a particle, though, is not affected by the dipole kick. The change in the closed orbit of a particle due to the dipole kick is negligible for small kicks but is large for sizeable kicks. This orbit change can easily be computed $[11,12]$. The orbit change has been verified and observed at the large electron positron (LEP) collider. In 1995, this orbit change eventually limited the luminosity that the LEP collider operated at with bunch trains [13].

The change in the closed orbit reference system due to the dipole kick must be taken into account in the simulation. A subtraction of the dipole kick is necessary to keep the particle in the correct reference system. The simulation code 

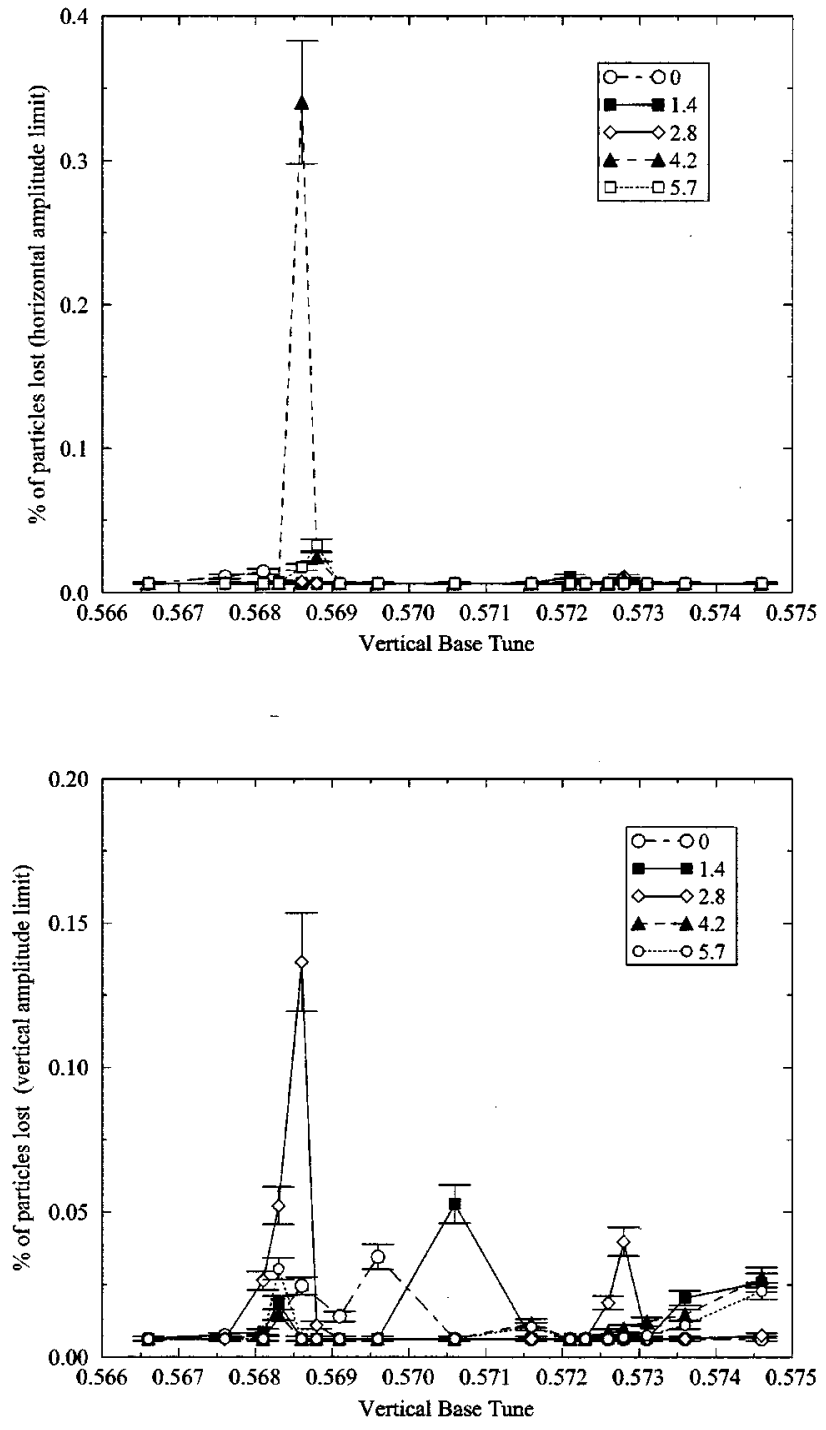

FIG. 11. Simulation of a $1 \times 1$ store measuring lost particles in a vertical tune scan. Lost particles in the top and bottom figure are defined with a horizontal and vertical amplitude limit, respectively. Each symbol represents a different beam separation at $B 0$ in units of $\sigma$.

subtracts the dipole kick contribution as shown

$$
\Delta x_{\text {total }}^{\prime}=\Delta x^{\prime}(y+d)-\Delta x^{\prime}(d) .
$$

It is assumed in this analysis that the particle's closed orbit is essentially stable and that no coherent dipole motion is driven by the beam-beam effect. A coherent dipole motion is excited when the coherent $\pi$ mode is close to a low order resonance; first and second order in the case of separated beam-beam kicks. Coherent dipole motion would result in a very fast particle loss and the $\pi$ mode would be very prominent in the tune spectra [13]. Such a coherent motion is therefore easy to detect and to avoid by an appropriate choice of the tune.

Figure 10 displays the simulated tune footprints for the antiproton distribution of measurement 2 of Fig. 8. Resonant effects are not shown in the figure; the footprint was ob- tained from simulation runs in a "resonant-free" region and overlaid on the proton tune of measurement 2. Each plot in the figure represents the tune footprint for four different beam separations.

Note that there is a shift in the cross hairs of Figs. 8 and 10. The proton tune in the simulation represents an unshifted proton tune; protons are not beam-beam tune shifted in a weak-strong model. The proton tune of Fig. 8 is a measurement of proton tune using Schottky detectors in the collider. These detectors have been found to measure the coherent motion of protons [14]. It is assumed that the proton tune measurement in the collider is representative of the tune of small amplitude protons. Since these protons are slightly beam-beam tune shifted in the collider, the proton tune shown in Fig. 8 must be tune shifted in the simulation in order to look at the proper resonant effects. The cross hair in Fig. 10 is shifted down and to the left by approximately 0.002 tune units to represent the unshifted proton tune of measurement 2 of Fig. 8 [4]. In effect, Fig. 10 is a qualitative picture of the initial tune spread of the antiproton distribution and indicates that resonant effects of the $7 Q_{x}$ resonance are observed in the head-on case and for a beam separation of $1.4 \sigma$. Resonant effects of the $\left(1 Q_{x}+6 Q_{y}\right)$ sum resonances are observed in measurement 2 for beam separations of $2.8 \sigma$ and $4.2 \sigma$.

Resonant effects were measured by monitoring the maximum amplitude reached by a particle during tracking. A particle was considered lost if it reached the tails of the Gaussian distribution; an amplitude limit of $3.5 \sigma$ was defined in both the horizontal and vertical plane. In a Gaussian distribution of particles, $99.95 \%$ are within a $3.5 \sigma$ amplitude range.

When simulating "lost particles," the absolute position of a particle at a given location in the ring is important. Since all amplitude particles in a particle distribution are kicked equally by the dipole kick, it is sufficient to add the orbit offset due to the dipole kick to the orbit offset measured during tracking. In the simulation runs presented, a maximum dipole kick of $4.2 \mu \mathrm{rad}$ occurred at $B 0$; the orbit offset due to this dipole contribution is $0.02 \sigma$ which is a negligible effect.

Lost particles measured in beam-beam simulations were compared to the particle losses of the measurement points of Fig. 9. A "simulated tune scan" measuring particle losses across the two seventh order sum resonances is seen in Fig. 11. The horizontal axis represents a variation in the vertical proton tune. The horizontal tune remained constant. Note that there is a shift in the proton tune in which resonant effects are observed when comparing the horizontal axis of Fig. 9 and Fig. 11. This again takes into account the beambeam tune shift of the small amplitude protons which was previously discussed.

The vertical axis of Fig. 11 is a measure of percentage $\mathcal{P}$ of particles lost from the particle distribution;

$$
\mathcal{P}=100 \times \sum_{b} \frac{w_{b}}{N_{b}} N_{L b}
$$

The factor $w$ represents a Gaussian weighting factor imposed on the initial antiproton distribution. Particles are binned 


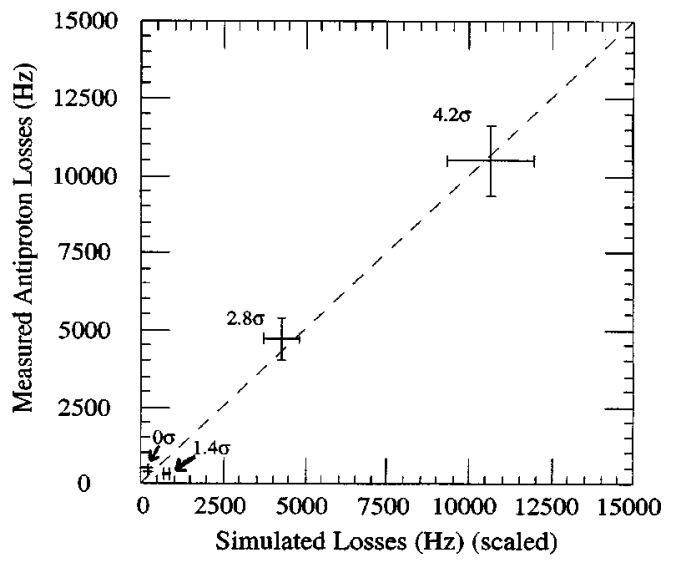

FIG. 12. A comparison of measured particle losses to simulated particle losses for measurement 2 of Fig. 6. Each data point represents a different transverse beam separation at $B 0$.

with bin index $b$ according to initial amplitude and the number of particles per bin, $N_{b}$, is weighted using a Gaussian dependence. The range of amplitudes in each bin is $1 \sigma$. The number of lost particles in each bin is $N_{L b}$.

Figure 11 displays particle losses across the particle tune spreads of measurements 1 and 2 . The figures display simulated lost particles with an imposed horizontal and vertical amplitude constraint, respectively. Each symbol in the plot represents a different transverse beam separation at $B 0$. For completeness, simulations were also run at a beam separation of $5.7 \sigma$, which corresponds to a $4 \sigma$ beam separation in both the horizontal and vertical planes. The resonant peaks in the tune scan occur at the $\left(1 Q_{x}+6 Q_{y}\right)$ resonance of measurement 2 .

A qualitative agreement between the simulation and the collider loss measurements of measurement 2 is observed. As is the case in the beam-beam experiment, simulated particle losses are low in the case of head-on collisions and for $1.4 \sigma$ separation. Losses are predicted to be largest at a beam separation of $4.2 \sigma$. The next largest particle loss is predicted at a beam separation of $2.8 \sigma$. Figure 12 summarizes a qualitative comparison of particle losses measured at the tune setting of measurement 2 in the collider to peak particle losses observed in the simulation. The error in measured particle losses is a reflection of the fluctuation of losses during the measurement; each error bar represents the standard deviation of particle losses over a four to five minute period.

A heuristic argument is used to estimate simulated particle losses. Simulated particle losses $\mathcal{L}$ in units of $(\mathrm{Hz})$ are obtained by

$$
\mathcal{L}=\left(\frac{\mathcal{P}}{100} N_{t}\right)\left(\frac{1}{\Delta t}\right) \mathcal{S}
$$

The total number of particles in the simulation is given by $N_{t}$. The time of tracking is $\Delta t$, where $\Delta t=$ (number of turns) $/ f_{\text {rev }}$. The parameter $\mathcal{S}$ is a constant scale factor used to make a comparison of loss measurements on a unit slope

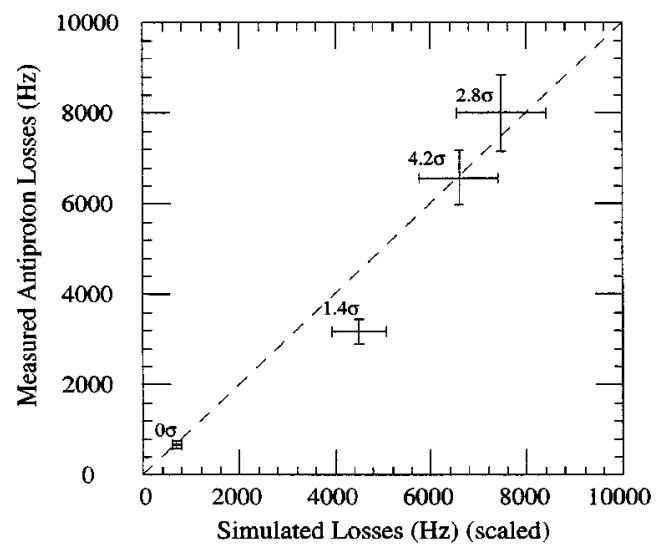

FIG. 13. A comparison of measured particle losses to simulated particle losses for measurement 3 of Fig. 6. Each data point represents a different transverse beam separation at $B 0$.

( $\mathcal{S}=400$ in this case). The uncertainty of simulated losses is the statistical variation of the number of lost particles in the simulation.

Figure 13 summarizes a comparison of simulation results with measurement 3. Particle losses driven by the $2 Q_{x}+5 Q_{y}$ resonance are measured. Simulated losses are observed to be greatest at a beam separation of $2.8 \sigma$. The qualitative comparison of measured losses at different beam separations is in agreement with the measured losses observed in the Fermilab collider.

\section{SUMMARY}

A comparison of beam-beam experiments with simulations led to a deeper understanding of the beam-beam interaction in the Tevatron collider. Experimental work determined that the beam-beam interaction is the predominant nonlinear driving term which drives 7 th order sum resonances in the Tevatron collider. Odd-ordered resonances are found to be driven in the presence of a transverse beam separation or when a crossing angle at an interaction point is present.

Simulated particle losses using a beam-beam model are shown to accurately predict relative magnitudes of beambeam resonant excitation at different transverse beam separations. At various tune settings, each representing a different resonant excitation, simulated particle losses as a function of beam separation were found to compare in a qualitative sense to measured losses in the Tevatron collider. With such a strong correlation between experiments and beam-beam simulations, many possibilities exist for future studies. One such possibility is using a beam-beam simulation to predict minimum beam separation criteria for future bunch configurations in the collider.

\section{ACKNOWLEDGMENTS}

The authors would like to thank the support given by Fermilab through the Joint University-Fermilab Doctoral Program in Accelerator Physics. Fermilab is operated by the Universities Research Association under Contract No. DEAC02-76CH00300 with the U.S. Department of Energy. 
[1] G. Dugan, in Proceedings of the 1989 IEEE Particle Accelerator Conference, pp. 426-430 (IEEE, Piscataway, NJ, 1989), pp. 426-430.

[2] L.R. Evans and J. Gareyte (unpublished).

[3] M. Meddahi, Ph.D. thesis, Université de Paris 7, 1991 (unpublished).

[4] D. Siergiej, Ph.D. thesis, University of New Mexico, 1995 [also Fermilab Note No. FN-627, 1995 (unpublished)].

[5] W. Herr, R. Schmidt, and M. Meddahi, CERN Report No. SL/91-05 (AP), 1991 (unpublished).

[6] S. Myers, in Proceedings of the Joint US-CERN School on Particle Accelerators, Lecture Notes in Physics Vol. 247 (Springer-Verlag, Berlin, 1985), pp. 176-237.

[7] M. Bassetti and G. Erskine, CERN Report No. IST-TH/80-06, 1980 (unpublished).
[8] A. Piwinski, IEEE Trans. Nucl. Sci. NS-24, 1408 (1977).

[9] A. Piwinski, IEEE Trans. Nucl. Sci. NS-32, 2240 (1977).

[10] K. Cornelis, W. Herr, and M. Meddahi, in Proceedings of the IEEE Particle Accelerator Conference, 1991 (IEEE, Piscataway, NJ, 1991), pp. 153-155.

[11] W. Herr, CERN Report No. SL/91-20 (AP), 1990 (unpublished) and LHC Note No. 149, 1990 (unpublished).

[12] E. Keil, CERN Report No. SL/94-76 (AP), 1994 (unpublished).

[13] W. Herr, CERN Report No. SL/95-08 (DI), 1995 (unpublished).

[14] G. Jackson, in Proceedings of the IEEE Particle Accelerator Conference, 1989 (IEEE, Piscataway, NJ, 1989), pp. 860-862. 